\title{
AOR
}

Selected Papers of \#AoIR2019: The $20^{\text {th }}$ Annual Conference of the Association of Internet Researchers Brisbane, Australia / 2-5 October 2019

\section{CALL ME MAYBE: Social expectations and trust associated with calling}

\author{
Cecilie Einarson Pérez \\ Telenor Group \\ Lorian Leong \\ Telenor Group
}

\section{Mobile communications in Scandinavia}

The accessibility of smartphone devices in mature markets like Sweden allow for an interesting exploration into youth smartphone cultures. Mobile devices insert themselves into the everyday lives of youth, from school hours, homework time, and leisure-time. Significant research has examined the emerging social norms surrounding mobile phone usage and youth culture, particularly focusing on telephony's core services from 1997-2005: mobile telephony and SMS in Norway (Ling, 2001) and youth culture among Norwegian teens (Laegran 2003). Recent research has focused on behaviours surrounding specific mobile communications applications, as they relate to areas of intimacy (Møller and Nebeling 2018), self-presentation and stigma (leong 2016), and personal expression (Fortunati 2005). Yang et al examined transitioning from textual to mobile calling as progressive mediums indicative of intimacy (2014). This research focuses on the cusp of communication through traditional mobiles (telephony and SMS) and internet apps. Although both involve voice and text, informants revealed distinctions between traditional and internet communications, establishing hierarchies in relation to intimacy and functionality, and sentiments around types of voice and text communication mediums. Crucially, this research delves into the level of trust in communications mediums and interpersonal relationships. This analysis examines how people communicate with each other based on perceived levels of trust and intimacy, and how technology becomes implicated and configured in those new systems of trust.

\section{Methods}

Suggested Citation (APA): Lastname, Firstinitial. (2019, October 2-5). Paper title. Paper presented at AolR 2019: The $20^{\text {th }}$ Annual Conference of the Association of Internet Researchers. Brisbane, Australia: AolR. Retrieved from http://spir.aoir.org. 
This paper draws on qualitative research conducted in Stockholm from 2016-2017 with 47 young people between the ages of 12- 22. Both individuals and groups with strong social ties were recruited for the study. All participants took part in semi-structured indepth interviews, and participants recruited as part of a group also took part in semistructured focus groups. In addition to reducing the imbalance of power between the interviewer and interviewee (Montell 1999), focus groups with some of the informants' peer groups confirmed norms individual informants expressed in 1:1 interviews as norms in practice. This research was conducted as part of a greater study intended for a division of Telenor Group examining behaviors and attitudes relating to communication among youth in Sweden.

\section{Judgement call: don't call us, we'll call you}

Impromptu voice calls via telephony marked two distinct user groups: strangers and strong ties. Informants discussed similarly opposing views of traditional telephony, as a medium for both communication with strangers (government services, utility companies), becoming part of what Ling calls the "broader social metabolism" (Ling 2012) and for very close friends.

For almost all informants, the fear, annoyance, and uncertainty of telemarketers and unknown numbers became a recurring theme. Telephony was also increasingly perceived by informants as a medium where strangers and telemarketers would reach them. Bertel (2013) notes Danish teens' trust in online maps that verification is not needed, suggesting a level of "taken for grantedness" (Ling 2012). Telephony, one of the older forms of technologically-mediated voice communications, among proved the opposite. Informant attitudes pointed to a decay of trust in the medium of telephony (not necessarily voice calling), one so easily abused by unknown callers. To manage this, the majority of informants used the number identification app TrueCaller or searched the number in online phone directories afterwards. This was all done to create a better sense of security in connection to receiving calls.

It was commonly understood that impromptu telephony calling from known contacts was one of urgency. There was an implicit trust among younger users that telephony was used for urgent situations, often deemed "serious" by informants. Calls from both strong and weak ties were perceived as urgent calls (barring few and exceptional cases for whom calling had become the norm), where weaker ties calling indicated something even more grave due to the abnormal nature of the behaviour. All contacts were therefore entrusted not to abuse this line of communication. For impromptu, synchronous communication of less severity, users were expected to use other communications apps like Snapchat.

\section{Who calls the shots?}

Considered an intrusive form of communication phone numbers were not shared amongst weak ties/acquaintances, and instead reserved for close friends and family members whom were trusted to know of and to adhere to these codes. As noted by Boase and Wellman (2006) synchronous communication can be perceived as 
demanding, leaving the respondent little flexibility to reply at their own convenience. However, intimate friends can slowly chip away at these established codes until new ingroup standards are created, like the case with Henrik's friend Johan:

"In the beginning, it felt strange when Johan called. It was like, 'What has happened now?' It must be something serious, but now we're used to it. He never sends messages."

Here Henrik illustrates Johan's breaking of two social codes: impromptu calling for serious matters and not sending messages before social calls.

Scheduled voice calls were coordinated through text first. This mitigated the urgency nature of impromptu telephony, indicating the motivation (and lower level of importance). Shorter phone calls were used for micro-coordination purposes to negotiate time and space, as first noted by Ling (2001) usually if texting became too cumbersome. However, they were understood as expected calls given the context of existing textual communication, thus maintaining social codes.

While informants had shared expectations surrounding general norms of functionality of calling, individuals expected their wider network to have specific time-based and spacebased knowledge of their schedules as part of their norms. Contacts ought to know their own individual rules and are trusted to adhere and abide by them, including convenient times to call. While individuals can eventually accept aberrant and "anti"social behaviour, acceptance is premised upon high levels of established intimacy. 


\section{References}

Bertel, Troels Fibæk. 2013. "“It's like I trust it so much that I don't really check where it is I'm going before I leave": Informational uses of smartphones among Danish youth." Mobile Media \& Communication 1 (3): 299- 313.

Fortunati, Leopoldina. 2005. "Mobile Telephone and the Presentation of Self." In Mobile Communications: Re-negotiation of the Social Sphere, edited by Rich Ling and Per E. Pedersen, 203-218. London: Springer-Verla London Limited.

Laegran, Anne Sofie. 2003. "Escape Vehicles? The Internet and the Automobile in a LocalGlobal Intersection." In How Users Matter The Co-Construction of Users and Technologies, edited by Nelly Oudshoorn and Trevor Pinch, 81-102. Cambridge: The MIT Press.

Ling, Rich. 2012. Taken for Grantedness: The Embedding of Mobile Communication into Society. Cambridge, MA: MIT Press.

—. 2001. "The diffusion of mobile telephony among Norwegian teens: A report from after the revolution." Presented at ICUST. Paris, France.

Møller, Kristian, and Michael Nebeling. 2018. "Bleeding boundaries : Domesticating gay hookup apps." In Mediated Intimacies: Connectivities, Relationalities, Proximities, by Michael Nebeling Petersen, Katherine Harrison, Tobias Raun Rikke Andreassen, pp. 208223. London: Routledge.

Montell, Francis. 1999. "Focus group interviews: A new feminist method." NWSA Journal 11 (1): 44- 71.

Wellman, Barry, and Jeffrey Boase. 2006. "Personal relationships: On and off the Internet." In The Cambridge Handbook of Personal Relationships, by Perlman D and Vangelisti AL, 709-723. New York: Cambridge University Press.

Yang, Chia-chen, B Bradford Brown, and Michael T Braun. 2014. "From Facebook to cell calls: Layers of electronic intimacy in college students' interpersonal relationships." New Media \& Society 16 (1): 5-23. 